\title{
Retracted: Histopathological Correlation between Prostatic Adenocarcinoma in Transrectal Ultrasound Guided Biopsies and Radical Prostatectomy Specimens
}

\author{
Mohammad Faisal' , Hina Tariq ${ }^{2}$ \\ 'Department of Histopathology, Sharif Medical and Dental College, Jati Umra, Lahore, Pakistan, \\ ${ }^{2}$ Department of Histopathology, Army Medical College, Rawalpindi, Pakistan
}

Received: 14 October 2019/Accepted: 12 December 2019

\section{OPEN ACCESS \\ Correspondence: Dr. Hina Tariq, Department of Histopathology, Army Medical College, Rawalpindi, Pakistan. Email: dr.hina2013@gmail.com}

Citation: Faisal $\mathrm{M}$ and Tariq $\mathrm{H}$. Retracted: Histopathological correlation between prostatic adenocarcinoma in transrectal ultrasound guided biopsies and radical prostatectomy specimens. J Cancer Allied Spec [Internet]. 2020 Jan.6; 6(1):e1002957. https://doi. org/10.37029/jcas.v6i1.299

Copyright: (c) 2020 Faisal and Tariq. This is an open access article distributed under the terms of the Creative Commons Attribution License, which permits unrestricted use, distribution, and reproduction in any medium, provided the original author and source are credited.

Funding: Nill.

Competing interests: Nill.

\begin{abstract}
Introduction: Differences in Gleason grade in transrectal ultrasonography (TRUS) biopsies and radical prostatectomy (RP) specimens are well documented in literature. Keeping in view the limitations of Gleason grading system, Epstein $\mathrm{Jl}$ grade group system was introduced. Various other parameters also have a significant role in predicting the pathological stage, extraprostatic extension, status of surgical margins and metastatic disease in regional lymph nodes. RP is performed at limited centres in Pakistan. Till date, no comparison of the histopathological findings in 12-core TRUS and RP specimens had been performed at the national level. Our study is aimed at generating local data in this context. Materials and Methods: This was a crosssectional study and non-probability consecutive sampling was performed. It was conducted at Histopathology Department, Shifa International Hospital, Islamabad, from January 2008 to December 2014. Gleason scores of 20 RP specimens were compared to Gleason scores of TRUS biopsies of same patients. Concordance in Gleason score and grade groups with laterality, perineural invasion was also studied. Results: Out of 20 RP cases, $40 \%$ ( $n=$ $8)$ had a Gleason score of $6,30 \%(n=6)$ had score $7,20 \%(n=4)$ had score 8 and $15 \%(n=3)$ had score 9. Compared to the TRUS biopsy, RP Gleason score was concordant in 11 cases (55\%), higher in 7 cases (35\%) and lower in 2 cases (10\%). TRUS involvement was unilateral in 10 cases (50\%) and bilateral in 10 cases (50\%). However, bilateral involvement of RP specimen was seen in 14 cases $(70 \%)$ and unilateral in 6 cases (30\%). Thus, better tumour yield was observed in RP specimens i.e., bilateral involvement in RP specimens was found in additional 5 cases (25\%). Perineural involvement was higher in RP specimen i.e., 12 cases (60\%), compared to 5 cases (25\%) in TRUS biopsies. Its concordance was significantly higher in those with Gleason score of equal to or more than 7 (83\%) and low in score less than score 7 (17\%). Conclusion: When comparing RP to initial TRUS biopsies, Gleason score was upgraded in $35 \%$ and downgraded in $10 \%$ of cases. Bilateral involvement in $25 \%$ of cases of RP specimens was underestimated as unilateral involvement in TRUS biopsies. Perineural involvement with high Gleason score was also seen.
\end{abstract}

Key words: Adenocarcinoma, biopsy, Gleason score, prostate cancer, radical prostatectomy, transrectal ultrasonography 


\section{Introduction}

Gleason grade importance is known for several decades in prostatic adenocarcinoma. ${ }^{[1]}$ It is accepted as an important parameter in decisionmaking of therapy. Grade 8 or above is a strong predictive of lymph node involvement. In such cases, pelvic lymphadenectomy or frozen section before radical prostatectomy (RP) can be planned. ${ }^{[2]}$

Gleason grading system is solely based on the architecture of tumour cells. Prediction of Gleason score in RP specimens by needle core biopsies improves with 18-gauge needle biopsies and more number of cores. Correlation is still not ideal in all cases and undergrading is a particular problem, in up to $58 \%$ of cases. ${ }^{[3]}$

Differences in Gleason grade in transrectal ultrasonography (TRUS) biopsies and RP specimens are well documented in literature. In addition to the above-mentioned reasons, another important area of difficulty was to quantify percentages of Gleason pattern Grades 3 and 4 when they are admixed. It is important to make a distinction between them. Gleason scores $3+4$ and $4+3$ are clinically distinct. ${ }^{[4]}$ There are also differences in tumour laterality as predicted by TRUS biopsy. Significant discordance is observed in low-grade tumour as compared to high-grade tumours. It is important because a high-grade tumour is associated with more extensive disease in the prostate and has significant potential to spread outside the prostate..$^{[1]}$ In cases with low-grade disease at initial TRUS biopsies, it had been recommended that treating surgeon should take into account the other clinical parameters and limitations of TRUS biopsy in assessing the Gleason score. ${ }^{[5]}$

Keeping in view the limitations of Gleason grading system, Epstein Jl grade group system was introduced. It is now formerly accepted and published in the World Health Organization Classification of Tumours of the Urinary System and Male Genital Organs $4^{\text {th }}$ Edition, Volume 8 in 2016. ${ }^{[6]}$ Univariate and multivariate statistical analyses were performed to assess the grade grouping system to estimate its implication and were found to be independently related with increased risk of prostate cancer-related mortality within 15 years of follow-up. Patients with grade groups 3-5 significantly progressed to cancerspecific death. ${ }^{[7]}$

In the grade group system, score begins from $3+3=6$. Patterns defined as 3 and 4 are more clearly defined. ${ }^{[6]}$ Total Gleason score 6 which is grade Group 1 is considered a relatively indolent disease. Total Gleason scores $3+4$ and $4+3$ are segregated in grade Groups 2 and 3 as they have different prognosis while total Gleason scores 8 and 9-10 are grouped under grade Groups 4 and 5 , respectively, which are associated with worse prognosis. ${ }^{[6]}$

Clinical management of prostate cancer has evolved significantly so has the grading system of prostate carcinoma. These changes in grading will facilitate the clinicians to better deal with these patients. At present, Gleason score and grade groups are to be reported simultaneously in each report to make the pathologists and clinicians well versed with this new system. With time, they will be able to directly use grade group without mentioning Gleason score. ${ }^{[6]}$

Various other parameters also have a significant role in predicting the pathological stage, extraprostatic extension, status of surgical margins and metastatic disease in regional lymph nodes. These include the percentage of tumour in TRUS biopsy and perineural invasion (PNI). ${ }^{[8]}$ If tumour is $>50 \%$ in any core or $>50 \%$ of TRUS biopsy, then there is an increased possibility of detecting high-risk pathological findings in RP specimens..$^{[9]}$ Extended prostate biopsy scheme had significantly improved the precision and correlation between Gleason score in TRUS biopsy and RP. ${ }^{[10]}$

Tumour volume estimate is also quite challenging, particularly in the setting of multiple positive sites. It is critical to decide which of the data is more predictive of final tumour volume and hence in clinical decision-making. ${ }^{[4]}$ 
RP is performed at limited centres in Pakistan. Till date, no comparison of the histopathological findings in 12-core TRUS and RP specimens had been performed at the national level. Our study is aimed at generating local data in this context. The objective of this study was to determine the correlation between histopathological features of prostatic adenocarcinoma in transurethral ultrasound-guided (TRUS) biopsy with RP specimens.

\section{Materials and Methods}

This study analysed 20 patients who underwent RP with or without neoadjuvant hormonal therapy (NHT) for prostate cancer between January 2008 and June 2014 at Shifa International Hospital, Islamabad. All patients irrespective of age were included in the study. All patients had positive TRUS core biopsy. Gleason grading system and Epstein JI grade grouping were utilised for grading. ${ }^{[6]}$ Tumour volume in a positive TRUS biopsy was defined as the percentage of areas of biopsy cores involved by carcinoma and was visually estimated. $\mathrm{RP}$ specimens were examined and processed according to standardised protocols. Gleason score, laterality, tumour volume, $\mathrm{PNI}$, extraprostatic extension, seminal vesicle invasion, status of lymph nodes if dissected and final pathological staging were also recorded.

Gleason scores of TRUS biopsies were compared with the respective Gleason scores of RP specimens. Concordance in Gleason score, laterality, tumour volume and PNI on TRUS biopsies with Gleason score, laterality, tumour volume, extraprostatic extension and pathological staging was performed.

Results were analysed by statistical software SPSS version 20. Mean was calculated for age and PSA. Frequency and percentages were calculated for primary Gleason grade, secondary Gleason grade, total Gleason score, grade group, tumour volume, $\mathrm{PNI}$, laterality, extraprostatic extension and pathological staging in both specimens. Concordance was performed for Gleason score, grade group and tumour volume, PNI of TRUS biopsies with Gleason score, grade group, tumour volume, PNI, extraprostatic extension, surgical margins and pathological staging of RP specimens.

\section{Results}

Out of 20 RP cases, $40 \%(n=8)$ had a Gleason score of $6,30 \%(n=6)$ had score $7,20 \%(n=4)$ had score 8 and $15 \%(n=3)$ had score 9 [Figure 1]. Compared to the TRUS biopsy, RP Gleason score was concordant in 55\%, higher in $35 \%$ and lower in $10 \%$. One of the cases could not be graded due to scanty nature of tumour after NHT [Figures 2 and 3].

Eight cases fall in grade Group 1, 4 in grade Group 2, 2 in grade Group 3, 4 in grade Group 4 and 3 in grade Group 5. Unilateral involvement was initially reported in 10 cases on TRUS biopsies. However, during analysis of RP specimens, four cases were

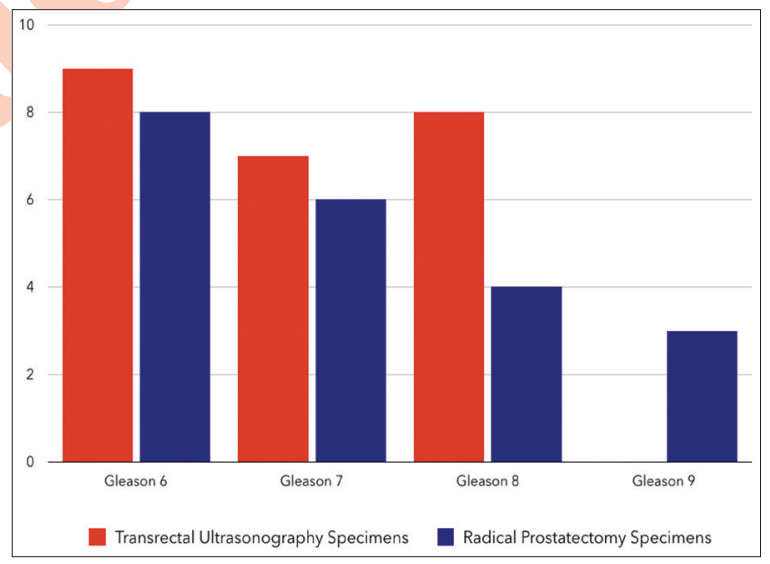

Figure 1: Comparison of Gleason score in transrectal ultrasonography and radical prostatectomy specimens

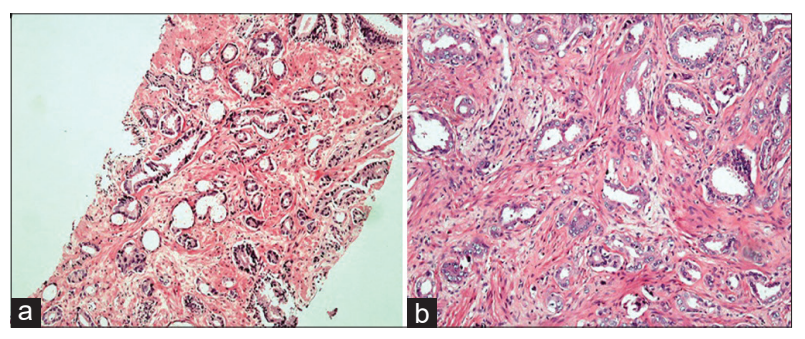

Figure 2: (a) Transrectal ultrasonography showing small glands lined by cuboidal to columnar epithelium and perineural invasion, grade Group 1. (b) Radical prostatectomy specimen of same patient showing grade Group 1 
upstaged because of bilateral involvement. All 10 cases with bilateral tumour on TRUS biopsies showed similar findings in RP specimens.

PNI was higher in RP specimen i.e., in 60\% $(n=12)$ of cases, compared to $25 \%(n=5)$ in TRUS biopsies with high concordance in those with Gleason score of $\geq 7$ ( $83 \%)$.

EPE was seen in $35 \%(n=7)$. About $43 \%(n=3)$ of these had Gleason score of 8 , while both scores 9 and 7 were $28 \%(n=2)$ [Figure 5]. All of these cases had $\mathrm{PNI}$.

Pathological tumour stage showed concordance with Gleason score, PNI and EPE. Out of eight cases of Gleason score 6, one was PT1 and seven were pT2. Majority of cases with Gleason score 7 and above i.e., 10 out 12 (40\%) were pT3 while 1 case of Gleason score 7 and 8 fall in pT2. All three cases of Gleason score 9 were pT3. All T3 cases showed EPE.

NHT was given in $40 \%(n=8)$ and showed disease confinement to organ in $87 \%$ with negative surgical margins.

\section{Discussion}

RP is the usual treatment of high-grade prostatic adenocarcinoma. Various factors such as Gleason grade group, Gleason score, pathologic stage, margin status, $\mathrm{PNI}$ and tumour volume predict the long-term prognosis. ${ }^{[11]}$

Prostate needle biopsy findings provide key information to plan treatment. ${ }^{[9]}$ Sextant biopsy schemes were traditionally used to predict the above-mentioned important prognostic features in RP specimens. However, these had poor correlation with prostatectomy Gleason scores. ${ }^{[11]}$ Extended 12-core biopsy has significantly improved prostate cancer detection and precision of Gleason score. ${ }^{[11,12]}$ Various studies have highlighted different reasons for significant discordance between the Gleason score in core biopsies and RP specimens. These include pathological interpretation bias and

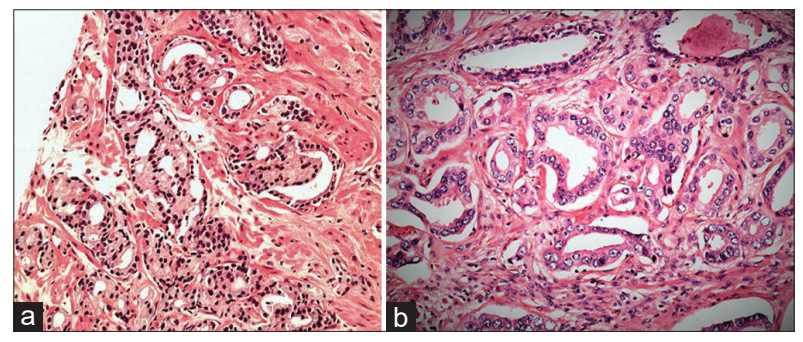

Figure 3: (a) Transrectal ultrasonography biopsy of a patient showed fused glands in majority of cores and some spaced glands were also seen. Grade Group 3 was assigned. (b) Radical prostatectomy specimen of same patient showed predominantly Gleason score 3, final grade group was 2

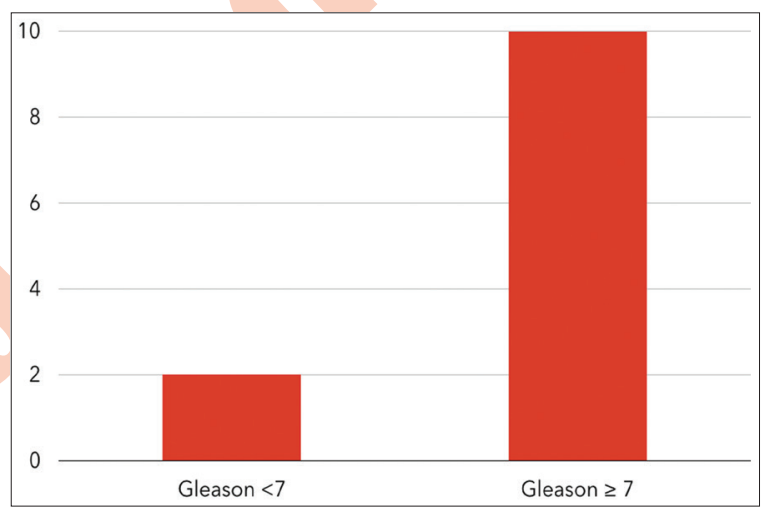

Figure 4: Perineural invasion in transrectal ultrasonography and radical prostatectomy specimens

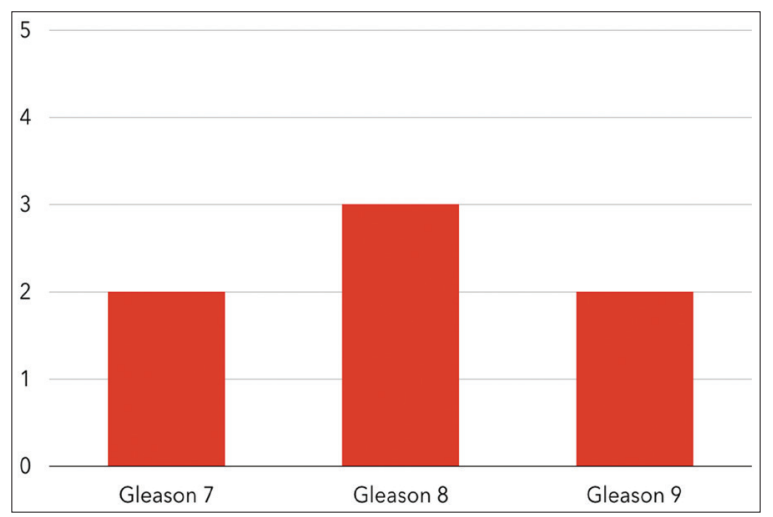

Figure 5: Results of extracapsular involvement in various Gleason scores

sampling effects like number of core biopsies. ${ }^{[5,12]}$ Hence, it is important for clinicians to consider various other clinical parameters and limitations of core biopsy. ${ }^{[5]}$ 
Undergrading of tumour in the needle biopsy, with a higher Gleason score in the matched RP specimens, is the most common problem. Prior studies have reported undergrading in $27-72 \%$ of cases [Table 1]. ${ }^{[2,5,9,13,14]}$ They mentioned that most of such cases were of low grade and probable reasons attributed to this were number of biopsy cores, procedure, prostate volume and lack of specialist expertise at the reporting centre. ${ }^{[2,5,9,13,14]}$ In our study, $35 \%$ of cases showed higher grade in RP specimens. In a study by Pinthus et al., approximately half of tumours graded Gleason score 6 at biopsy were found to have Gleason score 7 in RP specimens. Upgraded Gleason score 6-7 tumours have outcomes similar to those of genuine Gleason score 7 carcinoma. Hence, authors recommended that for Gleason score 6 carcinomas in prostate biopsy, clinicians should judge the overall likelihood of tumour upgrading as well as specific patient characteristics, such as prostate-specific antigen and the percentage of tumour (tumour volume) in the prostate biopsy when contemplating options that are optimised for low-grade tumours including observation or brachytherapy. ${ }^{[15]}$

We found lower grade in RP in 10\% of total cases. Downgrading in Gleason score has been previously documented in literature, the percentage of cases

\section{Table 1: Summary of prior literature examining the change in gradation of tumour between radical prostatectomy specimens and TRUS biopsies}

\begin{tabular}{l|c|c}
\hline Study & $\begin{array}{c}\text { Percentage of } \\
\text { cases that were } \\
\text { upgraded }\end{array}$ & $\begin{array}{c}\text { Percentage of } \\
\text { cases that were } \\
\text { downgraded }\end{array}$ \\
\hline Present study & 35 & 10 \\
\hline $\begin{array}{l}\text { Evan et al. } \\
(2016)^{[5]}\end{array}$ & 31.5 & 14.3 \\
\hline $\begin{array}{l}\text { Moon et al. } \\
(2010)^{[13]}\end{array}$ & 53 & - \\
\hline $\begin{array}{l}\text { Cohen et al. } \\
(2008)^{[14]}\end{array}$ & 36 & 5 \\
\hline $\begin{array}{l}\text { Zam et al. } \\
(2008)^{[9]}\end{array}$ & 27 & 81.8 \\
\hline $\begin{array}{l}\text { Cam et al. } \\
(2002)^{[2]}\end{array}$ & 71.7 & \\
\hline
\end{tabular}

varies from $5 \%$ to $82 \%$ [Table 1]. ${ }^{[2,5,9,14]}$ Similarly, in a meta-analysis, up to $7 \%$ downgrading had been reported. ${ }^{[14]}$ Elabbady and Khedr made two groups in their study and compared TRUS-guided lateral sextant biopsy and TRUS 12-core biopsies. Former technique showed downgrading in $50 \%$ of cases while later technique showed downgrading in $14.8 \%$ of cases. This study concluded that the extended 12-core prostate biopsy is more reliable in both the detection of prostatic carcinoma and accuracy of Gleason score. ${ }^{[16]}$ A Camtosun and Gökçe also mentioned that the reason of significant differences in Gleason score in TRUS and RP specimens became more obvious with increasing age, high PSA levels and decrease in prostate volume. ${ }^{[17]}$

RP Gleason score was concordant in $55 \%$ of our TRUS biopsy cases. Few studies also showed accurate prediction of Gleason score in 28.7-85.2\% of the cases. ${ }^{[2,5,14,16]}$ Reason of this wide variation in lack of Gleason score precision was attributed to lack of specialist expertise of pathologist and number of core biopsies. ${ }^{[5,16]}$

Bilateral tumour was initially detected in 44 out of 100 cases. In 56 cases, tumour was found unilaterally in TRUS biopsies, but later 37 out of these showed bilateral prostate involvements. Hence, tumour upstaging was appreciated in RP specimen. ${ }^{[1]}$ Tumour laterality discordance along with Gleason score tumour volume was also reported in some other studies when TRUS findings were compared with RP specimens. ${ }^{[5]}$ Four of our cases also showed upstaging due to bilateral tumour out of 10 cases with unilateral tumour reporting on TRUS biopsy.

$\mathrm{PNI}$ is assessed routinely in TRUS biopsies and is relatively reproducible. Detection of perineural invasion does not require the use of any other ancillary technique. It is defined as prostate cancer around the nerve within perineural space. $\mathrm{PNI}$ is an important mechanism of spread of prostatic carcinoma in periprostatic tissue. Its detection in RP specimen has no significance; however, if it is found in TRUS biopsies then may alter treatment plan. This issue has been a matter of debate for a 
significant period. ${ }^{[18]}$ Many studies have associated it with extraprostatic extension and seminal vesicle invasion. ${ }^{[19]}$ Multivariate analysis also showed similar findings. ${ }^{[19]}$ There are few studies which did not find such association. Vargas et al. concluded that PNI is not an independent predictor of extraprostatic extension when PSA levels were included. O'Malley et al. were also unable to demonstrate that its detection of TRUS influences long-term tumourfree survival. ${ }^{[20]}$ We observed that 7 cases had EPE out of 10 cases which showed PNI.

In addition to the above-mentioned reasons for discrepancy in findings between TRUS and RP specimens, handling of radical specimens by different pathologists may be a reason. As some of the specimens were entirely submitted while in other cases, only representative sections were examined. Few centres are currently processing RP specimens, so few histopathologists are actually well versed with fine details of reporting. However, there is a hope that with the introduction of grade grouping and with increasing number of specimens in good institutions and with adequate training, the concordance between the specimens will improve in future.

There were differences observed in Gleason Grade between TRUS and RP. Nonetheless, TRUS biopsies appear to have the potential to aid in further management of the patients with prostate adenocarcinoma. This minimal invasive technique may help in establishing diagnosis and treatment planning in regions where the access to RP is limited. Additional studies are recommended to establish sensitivity and specificity of TRUS and to determine overall benefits of the Grade grouping.

\section{Conflicts of Interest}

The authors declare that there are no conflicts of interest.

\section{References}

1. Bulbul MA, El-Hout Y, Haddad M, Tawil A, Houjaij A, Bou Diab N, et al. Pathological correlation between needle biopsy and radical prostatectomy specimen in patients with localized prostate cancer. Can Urol Assoc J 2007;1:264-6.

2. Cam K, Yucel S, Turkeri L, Akdas A. Accuracy of transrectal ultrasound guided prostate biopsy: Histopathological correlation to matched prostatectomy specimens. Int J Urol 2002;9:257-60.

3. Poulos CK, Daggy JK, Cheng L. Preoperative prediction of Gleason grade in radical prostatectomy specimens: The influence of different Gleason grades from multiple positive biopsy sites. Mod Pathol 2005;18:228-34.

4. Poulos CK, Daggy JK, Cheng L. Prostate needle biopsies: Multiple variables are predictive of final tumor volume in radical prostatectomy specimens. Cancer 2004;101:527-32.

5. Evans SM, Patabendi Bandarage V, Kronborg C, Earnest A, Millar J, Clouston D. Gleason group concordance between biopsy and radical prostatectomy specimens: A cohort study from prostate cancer outcome registry-Victoria. Prostate Int 2016;4:145-51.

6. Kryvenko ON, Epstein JI. Prostate cancer grading: A decade after the 2005 modified Gleason grading system. Arch Pathol Lab Med 2016;140:1140-52.

7. Erickson A, Sandeman K, Lahdensuo K, Nordling S, Kallajoki M, Seikkula H, et al. New prostate cancer grade grouping system predicts survival after radical prostatectomy. Hum Pathol 2018;75:159-66.

8. Kryvenko ON, Diaz M, Meier FA, Ramineni M, Menon M, Gupta NS. Findings in 12-core transrectal ultrasound-guided prostate needle biopsy that predict more advanced cancer at prostatectomy: Analysis of 388 biopsy-prostatectomy pairs. Am J Clin Pathol 2012;137:739-46.

9. Zam NA, Tan PH, Sim HG, Lau WK, Yip SK, Cheng CW. Correlation between prostate needle biopsies and radical prostatectomy specimens: Can we predict pathological outcome? Pathology 2008;40:586-91.

10. Mian BM, Lehr DJ, Moore CK, Fisher HA, Kaufman RP Jr., Ross JS, et al. Role of prostate biopsy schemes in accurate prediction of Gleason scores. Urology 2006;67:379-83.

11. Srigley JR. Key issues in handling and reporting radical prostatectomy specimens. Arch Pathol Lab Med 2006;130:303-17.

12. King CR, Long JP. Prostate biopsy grading errors: A sampling problem? Int J Cancer 2000;90:326-30.

13. Moon SJ, Park SY, Lee TY. Predictive factors of Gleason score upgrading in localized and locally advanced prostate cancer diagnosed by prostate biopsy. Korean J Urol 2010;51:677-82.

14. Cohen MS, Hanley RS, Kurteva T, Ruthazer R, Silverman ML, Sorcini $A$, et al. Comparing the Gleason prostate biopsy and Gleason prostatectomy grading system: The Lahey Clinic Medical Center 
experience and an international meta-analysis. Eur Urol 2008;54:371-81.

15. Pinthus JH, Witkos M, Fleshner NE, Sweet J, Evans A, Jewett MA, et al. Prostate cancers scored as Gleason 6 on prostate biopsy are frequently Gleason 7 tumors at radical prostatectomy: Implication on outcome. J Urol 2006;176:979-84.

16. Elabbady AA, Khedr MM. Extended 12-core prostate biopsy increases both the detection of prostate cancer and the accuracy of Gleason score. Eur Urol 2006;49:49-53.

17. Camtosun A, Gökçe H. Comparison of prostate biopsy pathology and radical prostatectomy pathologies. Dicle Tıp Derg 2019;46:133-9.

18. Potter SR, Partin AW. The significance of perineural invasion found on needle biopsy of the prostate: Implications for definitive therapy. Rev Urol 2000;2:87.
19. Billis A, Quintal MM, Meirelles L, Freitas LL, Magna LA, Ferreira U. Does tumor extent on needle prostatic biopsies influence the value of perineural invasion to predict pathologic stage $>$ T2 in radical prostatectomies? Int Braz J Urol 2010;36:439-49.

20. O'Malley KJ, Pound CR, Walsh PC, Epstein JI, Partin AW. Influence of biopsy perineural invasion on long-term biochemical disease-free survival after radical prostatectomy. Urology 2002;59:85-90.

\section{Authorship Contributions}

Conceived and designed the analysis: MF; Collected the data: MF; Contributed data or analysis tools: MF, HT; Performed the analysis: HT; Wrote the paper: HT. 